\title{
Professional sexual abuse in mental health services: Capturing practitioner views of a contemporary corruption of care
}

\author{
Janet Melville-Wiseman ${ }^{1}$
}

\begin{abstract}
This paper focuses on practitioner views of professional sexual abuse in mental health services in the UK. In spite of high profile cases, and changes to the law and regulation of mental health professionals in recent years, it remains a significant problem. It also presents particular challenges for colleagues and managers of services when incidents are reported or come to light through everyday practice. To date it has received little attention in research other than from within psychological therapies. This study seeks to provide a counter balance to those approaches by taking a social inequalities perspective and focusing on systemic and institutional dimensions. It draws comparisons with Wardaugh and Wilding's (1993) theory of the 'corruption of care' and argues that even when mental health care is provided in the community it can still become corrupted. The paper presents the findings of a small scale study of practitioner perspectives on the subject. The findings tentatively suggest that the problem is endemic but hidden by ineffective management responses. It argues for new approaches to selection, training and support for professionals that include counter intuitive approaches to policing colleagues and institutional cultures.
\end{abstract}

Keywords: sexual abuse; adult safeguarding; professional abuse; boundary violations; professional misconduct

\section{Principal Lecturer in Social Work, Canterbury Christ Church University}

Addres for correspondence: Canterbury Christ Church University, North Holmes Road, Canterbury CT112U, UK. janet.melville-wiseman@canterbury.ac.uk

Date of publication (online): 20th August 2012 


\section{Introduction}

Professional sexual abuse in mental health services (PSAMHS) has received surprisingly little attention in the literature on safeguarding; the quality of mental health services; or the regulation, education and training of health and social care professionals. Much of the literature on the subject has been generated in the US with a focus on sexual boundary violations in psychotherapy (Schoener, 1995; Melville-Wiseman, 2008). However, it remains an issue of considerable concern to health and social care regulators in the UK including the General Social Care Council (GSCC) which with "which was the regulatory body for social workers in England until August 2012. What they euphemistically refer to as 'inappropriate relationships' accounted for $40 \%$ of the social workers who were removed from the GSCC register between 2003 and 2008 (GSCC, 2008). In response to this the GSCC commissioned research into professional boundaries (Doel et al, 2009) and recently produced additional guidance for social work practitioners (GSCC, 2011). However both the research reports and the guidance focused on general boundary questions rather than on the distinctively challenging problem of preventing or responding to sexual boundary violations. This paper draws on the second phase of a research project and aims to capture the views of a range of stakeholders on PSAMHS. The first phase of the study was an instrumental case study of one woman's experience of PSAMHS. Drawing on Wardaugh and Wilding's concept of the 'corruption of care' written in the aftermath of abuse scandals in care homes in the 1980s (Wardaugh and Wilding,1993) it argues that PSAMHS is also an institutional scandal that will not be prevented by simply focusing on the individual psychopathology of victims, the ejection of perpetrators as a 'few rotten apples' from professions or simply by an increase in regulatory or legal prohibitions. It is a difficult subject to think about and a difficult issue to speak about.

For the purpose of this paper PSAMHS is defined as a situation where a professional is working with a person who is vulnerable by virtue of a mental health need and through that professional relationship engages in some form of sexual contact. Defining what is meant by a professional, a person vulnerable through mental health needs or where sexual contact may begin presents many challenges but for clarity this paper focuses on situations that are unambiguously wrong and harmful to victims. Such scenarios constitute a criminal offence under the UK Sexual Offences Act 2003 sections 38-44; a civil offence under the Mental Health Act 1983/2007 Section 127 and are breaches of all regulated health and social care professional codes of conduct, practice or ethics. It is also proscribed within multi agency policies on safeguarding vulnerable people (Department of Health, 2000).

For clarity, this paper defines the mental health system as in-patient facilities, residential care homes, nursing homes, day, sessional or domiciliary care provided by statutory, private or voluntary agencies. A professional is a psychiatrist, psychologist, psychotherapist, psychiatric or mental health nurse, occupational therapist, social 
worker, care worker, counsellor, clergy person, voluntary worker or student who offers a service. The term 'client' is used for brevity but this is not intended to discount the different terms people who use mental health services would choose or the different terms used within different health and social care professions. The term 'victim' is also used when referring to someone who has been abused but again this is not meant to discount the views of some who move from victim to survivor and sometimes activist following PSAMHS.

\section{Mapping professional sexual abuse in mental health services}

PSAMHS appears to be a subject that is difficult to speak about and difficult to articulate in an established or consistent way. This is perhaps symptomatic of why it is a difficult phenomenon to deal with effectively when it happens. Inconsistency is apparent in the language of regulation, research literature and UK law and policy. As mentioned above, the GSCC used the term 'inappropriate relationships' (GSCC, 2009); the Nursing and Midwifery Council - the regulatory body for UK nurses, reports on sexual abuse of a patient or inappropriate relationships with patient (Nursing and Midwifery Council, 2011); and the General Medical Council (the regulatory body for doctors including psychiatrists in the UK) uses the term 'relationships with patients' (General Medical Council, 2009). However, none of these terms indicate that the phenomena may in fact be a criminal offence. Failing to recognise this in basic terminology has the potential to minimise the seriousness of the impact and can lead to ineffectual responses when such incidents come to light (Kennedy, 2002; Hesketh, 2003).

However, the subject has also received little vigorous attention in research literature in the UK in spite of a steady stream of literature emerging from the US from the 1960s onwards. Such research not only showed the extent of the problem there but also that victims were often irreparably damaged by the experience (Gartrell et al, 1987; Schoener, 1995). Early prevalence studies, even when reliant on self disclosure by professionals, found that between $12 \%$ and $16 \%$ of male professionals and between $2 \%$ and $3 \%$ of female professionals admitted sexually abusing their clients (Kardener et al, 1973; Holroyd and Brodsky, 1977; Pope et al, 1979; and Borys and Pope, 1989). These studies found no differences between mental health professionals although one study (Gechtman and Bouhoutsos, 1985) which surveyed 1000 social workers found the incidence amongst them to be 3.8\%. A later study of a random sample of 1000 clinical psychologists in the UK (Garrett and Davis, 1994) showed that of the 581 completed questionnaires returned, 3.6\% admitted sexual contact with a client. However, $22.7 \%$ of respondents had treated a client who had previously been sexually abused by a mental health professional 
such as a psychiatrist, nurse or social worker; and 38\% reported that they had known of a psychologist who had sexually abused a client through other sources. In the UK the National Patient Safety Agency, which was set up to monitor reports of adverse patient incidents in the National Health Service, reported on all aspects of patient safety on mental health wards including sexual safety (National Patient Safety Agency, 2006). The report is based on data gathered between November 2003 and September 2005 and found that there were 122 incidents of a sexual nature including sexual harassment, sexual assault and rape. Of the 19 reported incidents of alleged rape, 11 were allegations against staff. The agency does not gather data on whether these allegations of rape were reported to the police. In a similar survey, 44 mental health trusts were asked to give information on the number of sexual assaults reported to managers. In the three years from 2003 to 2006 there were 300 reported incidents. Of those, 76 (25.3\%) were perpetrated by staff (Jackson, 2006). Both of these surveys rely on incidents having been reported to managers so may not represent the extent of the problem.

It also appears to be the case that, as with other forms of sexual offending, once a professional sexually abuses a client they are unlikely to stop of their own accord (Bates and Brodsky, 1989; Benowitz, 1995). This has been borne out by one of the most high profile investigations in the UK involving two consultant psychiatrists, Kerr and Haslam, who between them sexually abused over 70 clients, in the same hospital, during a period of twenty years, before eventually being prosecuted (Pleming, 2005). However, in the Kerr and Haslam case they neither stopped of their own accord neither did their employers or colleagues take effective action when different victims of their abuse came to light (Pleming, 2005).

\section{The institutional context of professional sexual abuse}

In Wardaugh and Wilding's (1993) examination of what they termed the 'corruption of care' they sought to identify what it was about the nature of institutions which allowed abusive practices to take place within their confines. They drew on earlier work by Foucault (1977) and Goffman (1961) who had begun to identify the depersonalisation and dehumanisation of individuals in institutions. Their theory may be relevant in terms of our understanding of PSAMHS. Specific changes may happen to professionals when they work in services particularly where power imbalances flourish and where clients are routinely depersonalised through medical models that focus on diagnoses and treatment. Wardaugh and Wilding (1993) argue that within such systems professionals are inadvertently authorised to apply unusual moral imperatives in their relationships with people in their care. Inhibitions about mistreating a fellow human being or witnessing another mistreat a fellow human being, will not apply and abuse can flourish. If managers within such systems are 
overburdened, weak or non-existent chains of accountability can develop with resultant direct or indirect collusion with abusive practices on their part also (Wardaugh and Wilding, 1993; Cambridge, 1999; Colton, 2002).

\section{Social inequalities}

Having a diagnosis of mental ill health brings with it stigma, discrimination and social exclusion for clients (Repper and Perkins, 2003). Many workers in mental health services also have high rates of significant mental health needs (Hawton et al, 2002; Stanley et al, 2007), However, much of the research on relationships between the two has focused on how to make the relationship therapeutic (for example O'Brien, 2001); how to work in partnership with the client and their family or carer (for example Biehal, 2005); or how to make the relationship safe from the possibility of physical harm usually to the professional or the wider community (for example Hewitt, 2008). Whilst some of these dimensions may have an impact on the power differentials between the two, the characteristics of that relationship that are rooted in social inequality and power imbalances have gained far less attention (Williams,1996; Repper and Perkins, 2003). However, professional power, gender and age seem to be key dimensions of PSAMHS.

D'Addario (1977) found that primarily male professionals committed sexual offences against women clients; these professionals had a mean age of 43.5 years; they had been in practice for between 5 and 30 years and their victims were approximately 12-16 years younger than them. Holroyd and Brodsky (1977) found that most professionals who sexually abuse their clients do so repeatedly (median = 6; mean $=29$ times) and probably serially abuse unless stopped by effective intervention. Schoener et al (1984) undertook a gender profile of 250 cases. Of these 250 cases, $12 \%$ ( $n$ 30) involved a female professional sexually abusing a female victim; 3.2\% ( $n$ 8) was a male professional and male victim and $1.4 \%(n)$ a female professional and male victim. However, it was nearly always women who were the victims (95.2\%) and that same sex abuse between women was more common than same sex abuse between men (ratio 1:0.13). One conclusion from this might be that the power accorded to mental health professionals may be a more significant factor than the power related to gender.

In 2004, the UK mental health charity MIND published its Ward Watch Campaign findings which found that $18 \%$ of in-patients had experienced sexual harassment whilst an in-patient and $5 \%$ reported sexual assault. However, they also found that whilst $56 \%$ of those assaults or incidents of harassment were perpetrated by other patients, 31\% were perpetrated by ward staff (MIND, 2004). 


\section{Personal testimonies}

A significant contribution to our understanding of PSAMHS has been made by victims - either by publishing their own stories (Bates, 1989; Penfold, 1998; Russell, 1993; Wohlberg et al, 1999) or by participating in research or inquiries aimed at capturing their views (Peterson, 1992; Acker, 1995; Casemore, 2001; Pleming, 2005). Common themes from such testimonies include bewilderment and disbelief that their abuser could breach their trust (Bates, 1989, Russell, 1993; Acker, 1995); a period of grooming by the perpetrator whilst in the treatment setting (Wohlberg et al, 1999; Penfold,1998); a sense of shame and humiliation which makes it difficult to disclose similar to those associated with childhood abuse disclosure (Acker, 1995; Penfold, 1998); and a sense of fear about how the experience may affect their personal relationships (Penfold, 1998). Many victims are not able to disclose the abuse for many years (Wohlberg et al, 1999); and some speak of the sadness but also relief at finding other victims of the same professional who are then able to validate their testimony. (Casemore, 2001; Pleming, 2005).

\section{Lack of responses in services}

One of the major concerns raised by the Pleming report into the multiple sexual abuses perpetrated by former consultant psychiatrists Kerr and Haslam in the UK was the lack of effective intervention by managers and other professionals when whistle blowers came forward (Pleming, 2005). This was also the case in earlier studies in the US and elsewhere. For example, Fortune (1992) describes organisations 'shooting the messenger' when they do not want to deal with the main problem (Fortune, 1992 p. 120). A study in New Zealand (Agar and Read, 2002) found that even when sexual abuse, whether perpetrated by professionals or others, was disclosed by clients, many mental health professionals ignored it. Even when recorded in a case file, and even when significant risk factors were present, few treatment or prevention plans were made. A recent study into reporting of adult protection concerns showed that mental health services referred the fewest number of people (Cambridge et al, 2006). However, Brown and Keating (1998) found that mental health professionals showed an almost blasé approach to adult safeguarding, claiming that if they reported abuse they would be 'doing it all day' (Brown and Keating, 1998 p. 276. 


\section{Method}

This paper presents the second phase of a research project into PSAMHS. The first phase was an instrumental case study which took a systemic view of one woman's experience of being raped by her CPN (Stake, 1995). The woman has asked to be known as Alice. The second phase involved a small self selected sample of a group of professionals in mental health services, including safeguarding, mental health practice, education and training who were asked to comment on Alice's experiences and how they resonated with their own knowledge of PSAMHS.

The choice of case study as a method for the first phase aimed to provide a counterbalance to the largely survey driven studies of the past. This approach also supported the aim of capturing the voices of participants and the fine grain detail of an example of the phenomena. This means seeking to understand what it was like as opposed to uncovering generalisable data (Yin, 2009). The use of case study as a research method has its roots in the need to understand the social context of the human condition (Deegan, 2001; Yin, 2009) and has been found to be particularly useful in disciplines such as nursing, education and psychology (Bergen and While, 2000). As an instrumental case study meant it was used to understand the phenomena as opposed to the case itself being of significant concern (intrinsic) or where a number of examples are joined to understand a phenomena (collective) (Stake, 1994). In this study the design, or as Yin (2009) describes it, the 'tactic' (Yin, 2009 p.41) involved identifying multiple sources and types of data; systematic collection and management of data; establishing an audit trail of evidence; and through analysis of convergent or divergent data, building explanations (Yin, 2009). For the first phase sources and types of data included documentary and archival sources from Alice's past, and observations and interviews with people identified as having a personal or professional connection to the case. The research utilised a feminist emancipatory approach whereby Alice's story would not be objectified but rather her story told as close to her own words and experiences as possible (Grbich, 1999; Truman et al, 2000).

\section{Strengths and limitations of the method}

Telling the story is a significant and useful dimension of any research where the topic has previously been surrounded in a culture of silence or secrecy such as child abuse (Woodward, 2000) or the topic covered here. However, criticism of case study research includes the potential for subjectivity by both participants and researcher (Simons, 2009). In this example the subjective experiences were the main points of interest and the main vehicle for building explanations of PSAMHS in its social context (Stake, 1995). In addition a researcher reflective log was kept during data gathering and date analysis in order to identify how each stage developed. This 
also provided a further audit trail of the process of the data collection which adds rigour to this type of study where the boundaries of the case are not known prior to commencement (Jasper, 2005).

The second phase reported here utilised the 'real life' case vignette (Alice's story) to illicit the views of a range of stakeholders. The use of case vignettes in social research can provide a better test of views than a simple questionnaire or interview approach as it gives the informants a concrete example of the phenomena on which to base their responses (Alexander and Becker, 1978). However, it also gives the researcher the advantage of a constant factor across informants which is not related to their own direct experiences. This has been found to be particularly useful in research where informants may have a personal connection to a sensitive subject (Hughes and Huby, 2002).

The study deployed both a transactional and transformational approach by checking back with participants at each stage and giving as full an account as possible of the findings (Cho and Trent, 2006).

\section{Participants}

Participants were recruited by word of mouth from local mental health and other services. One participant was also a former user of mental health services; two had been sexually abused by a professional in a counselling situation, and one was also the former wife of a sexually abusive professional. It is not a representative sample and was likely to attract participants who had some concern about the topic. Two additional mental health practitioners considered participating but later declined citing fear of the repercussions for their work if their managers discovered that they had taken part in a research on this subject.

Data was gathered by individual semi-structured interviews which were electronically recorded and then transcribed. Participants were given key details of Alice's story as a real life case vignette and were asked to comment on her experiences but also on their experiences of PSAMHS and the social and institutional context in which it occurs.

\section{Data analysis}

Data analysis followed a process of intuitive interpretation of the data predicated on researcher assumptions inherent in the rationale for the study (Simons, 2009). This means that the study is not replicable but again the rationale was to illuminate details rather than find casual links. Researcher assumptions included that PSAMHS is both wrong and harmful; that the scope of that harm is currently under recognised; and that the wider institutional context may be significant. In addition, responsibility 
for keeping boundaries belongs to professionals not clients. This is because the individual and collective covenant professionals make with vulnerable people includes helping people who find it hard to maintain their own boundaries. The study therefore rejected any approach that could directly or indirectly lead to victim blaming such as looking for psychopathological explanations.

The process of developing an explanation of the data used an inductive and intuitive process to generate themes. This included an initial examination of transcripts of interviews, field notes, observations and reflections; the development of a working hypothesis and the identification of themes. Themes were then organised using thematic networks from basic themes to organising themes and then global themes (Attride-Stirling, 2001). The findings are presented under global themes.

\section{Case vignette: Alice's story}

Alice disclosed that seventeen years ago when she was in her 40s she was raped on two occasions by her Community Psychiatric Nurse (CPN). This happened in her own home on the two days following her discharge from the Accident and Emergency department of her local hospital. She had taken a life threatening overdose on the first anniversary of her husband's suicide. She had received mental health services for many years and had a diagnosis of depression. Her experiences of mental health services prior to this had been characterised by poor interpersonal boundaries from mainly nursing staff, such as taking her out for drinks, asking her to house and dog sit and asking her out to dances. Her husband's suicide was linked to Alice's admission to a psychiatric hospital the year before when he felt he could not cope with her absence from the family and the effect on her of further electro convulsive treatment. With the help of an advocate Alice reported her experiences to the police who undertook a criminal investigation. The alleged perpetrator was still working as a mental health nurse in a different part of the country. Although he admitted having sexual intercourse with a woman patient on two occasions he claimed it was consensual and he denied rape. The police determined they were unable to proceed with a criminal prosecution. However, the Nursing and Midwifery Council (the regulatory body for UK nurses) proceeded with a case under their Fitness to Practice process and the perpetrator was struck off the nursing register. With all such orders he would not be able to apply for readmission to the register for at least five years. In the course of the investigation it came to light that when he was a student nurse he had been warned for inappropriate behaviour towards women students but had been allowed to continue training. The investigations were also characterised by poor boundary holding including from the chair of the regulatory body hearing and the woman police officer who interviewed Alice. 


\section{Findings}

\section{Social inequality}

There was a strong view that within mental health services power imbalances are linked to professional orientation, with psychiatrists holding the most power closely followed by nurses. There was also a view that staff in junior positions or students on practice placements would have the greatest difficulty in having their concerns about abusive staff taken seriously, but would often show the greatest courage and persistence. Gender was also thought to be a factor in terms of choice of worker, including for vulnerable men, and that systems of assessing risk when home visiting do not take account of such relational risks. In fact clients are perceived as the only dangerous people.

I think it is still very much the case that the psychiatrists have the power - followed closely by the CPNs. (Community Psychiatric Nurse)

Home visiting is done where there is thought to be a need or, if the patient wants to be seen at home, rather than in the clinic for a variety of reasons. If there is thought to be any risk to the staff the person would be seen by two members of staff. I don't think we think in terms of risk to the patient by individual staff. ( Community Psychiatric Nurse)

There was also the view that mental health services do not support victims to challenge their treatment or care so are unlikely to be able to challenge an abusive professional. Victims can also be blamed for the behaviour of professionals. One participant, who was a victim and the former wife of a counsellor who abused his clients, felt blamed by his organisation for his behaviour.

I would never share this with my colleagues as I feel there is a culture of clients always being in the wrong and I would be seen as to blame for my abuse and also how my husband behaved. I felt blamed when he lost his job - I felt people in his organisation thought it was my fault he did this, that I was not a good enough wife. (Mental Health Social Worker)

\section{Institutional context}

With one exception each participant knew of at least one colleague who had sexually abused a client but most knew of several cases. They all agreed that it was unequivocally wrong; that their respective institutions had not dealt with it effectively; and that there were degrees of collusion. However, there was also a sense of shame and powerlessness about their colleagues' behaviour. 
I know on one occasion a male nursing assistant had a relationship with a female patient and another female colleague knew about it and did nothing. She was sacked as well because there was collusion. They all went out socialising together. (Community Psychiatric Nurse)

I have also known doctors touch people inappropriately. In the old days, you think more went on in the old asylums but I don't think so. There is just as much today if not more. (Community Psychiatric Nurse)

I have come across situations where members of staff have had relationships with patients. I am completely against it but some people say it is ok but I think it is disgusting. (Community Psychiatric Nurse)

I know of a nursing assistant who had a relationship with a young patient who was under section [of the Mental Health Act 1983]. Also a qualified female had an affair with a male patient and a student had an affair with a patient who was detained [under section of the Mental Health Act 1983]. They now have a baby. Because I knew this patient I was really worried about it and now she is in training [to be a nurse]. (Community Psychiatric Nurse)

Perhaps the reason [that services do not respond well] is that because boundaries are so endemically poor in the whole system, that everyone feels a sense of complacency. It is a cultural thing really. (University Lecturer)

It happens far more than people think. Speaking with colleagues, most people have known either a client who has experienced this sort of thing or known of a colleague who has been involved in inappropriate relationships. (Mental Health Social Worker)

\section{Effectiveness of current systems designed to prevent PSAMHS}

Participants all stated that they felt that Alice's abuser should never be permitted to reapply for registration as a nurse. Some had known colleagues who had been struck off the professional register for PSAMHS but had then gained employment in a care home. Some knew colleagues who were continuing to work in the same role where they had already abused a client. There was also a sense within systems designed to address PSAMHS that nothing could or would be done.

I had to investigate the case of a man who was living in a care home but who suddenly disappeared. He had gone to set up home with one of the care staff. We had an adult 
protection conference but it was thought that nothing could be done as the care worker left. (Mental Health Social Worker)

I had to assess a man under the Mental Health Act who suffers from bi-polar disorder and when I tried to arrange his admission I was told that he could not go to the local hospital as he would start 'stalking one of the nurses'. When I spoke with him about it he admitted that he would try and speak with this nurse as she was the mother of his three children. When I checked this out with the nursing staff it was true. They had met when he was a patient and she was a nurse on his ward... The nurse should be struck off or at least be made to go and work somewhere else so that he can still use his local services. (Mental Health Social Worker)

A common theme was a lack of awareness of what constitutes abuse. Also, a lack of awareness of the professional imperatives to report abusive colleagues and none had done so. Some participants did not know which policy or procedure would apply and none had received training in dealing with PSAMHS at either prequalifying level or as part of on-going professional development. Trainers in adult protection work commented on ambivalence amongst practitioners who often felt that reporting colleagues would interfere with the therapeutic ethos of the team. However, it also seemed to be a counter intuitive task for people who had come into mental health work to help people and that reporting a colleague seemed an uncaring thing to do.

If people are not clear about what they should do they tend to do nothing. That is one of the reasons why I became a training manager when I realised there was widespread ambivalence and not being sure what to do. 'We shouldn't really be doing this; and what about confidentiality; what about the therapeutic bond; she told me and asked me not to say anything'. (Adult Protection Trainer)

It is never thought that someone might be vulnerable from an attack from staff. I don't think I would know what policy covered that. Also, I have never covered anything about asking questions about histories of sexual abuse in any training. (Community Psychiatric Nurse)

\section{Hopeful signs of good practice}

Participants who had been able to acknowledge their own personal connection to abuse felt that they would and had been able to offer particular support to victims of PSAMHS. However, they were certain that they would not disclose their own history to a client but also that they would not share it with colleagues or supervisors. 
I guess there is something for me about knowing the pain and the agony of being silenced for all kinds of reasons. Now it is very important for me to help people find their own voice because I know what torment it caused me.' Voluntary Agency Worker

Because of my experiences, I know if one of my clients begins to disclose something about abuse I know that I can be an ally; I know the right questions to ask; and I know how to help them put it into words. I also know how to reassure them that I am going to believe them. (Mental Health Social Worker)

One participant presented a very human, and for her, an effective approach to boundary keeping.

Why would you want to have a relationship with a client? Some of them I don't even want to work with. Even worse why would you want to have a relationship with a nurse or doctor? Some of them I don't want to work with either. (Mental Health Social Worker)

\section{Discussion}

These tentative findings have captured the views of a small group of professionals working in mental health services. The study aimed to present arguments for a systemic or institutional perspective on PSAMHS as part of debates about prevention. Although small scale there appeared to be consensus from participants that PSAMHS is a significant problem that has substantial harmful impact. The study clearly demonstrates that current arrangements for dealing with PSAMHS are not effective in terms of prevention or effective in terms of prevention or managing impact when it comes to light, and we should be looking for what Schoener (1995) refers to as a 'plan B' (Schoener, 1995). Some participants were concerned that there may be degrees of collusion within services or lack of effective and transparent management responses similar to those identified as part of the 'corruption of care' in care home scandals (Wardaugh and Wilding 1993, Cambridge, 1999; Colton, 2002).

Power differentials between professionals may be significant both in terms of client vulnerability to particular professionals in the hierarchy but also as a barrier to colleague reporting (Agar and Read, 2002, Cambridge et al., 2006). If psychiatrists have the most power, followed closely by nurses, it is unlikely that they, in particular, would want to destabilize those structures by dealing openly with abusive colleagues. Although each participant had known of at least one case of PSAMHS, and that most of them had not been dealt with adequately, none had become a whistle blower possibly fearing the consequences for their own employment (Pleming, 2005). They may have also not known what to do, or it had become commonplace for it to happen, and commonplace for it to be ignored 
(Wardaugh and Wilding, 1993; Brown and Keating, 1998; Cambridge et al., 2006), However, examples cited included instances of criminal behavior and where other vulnerable service users remained at risk (Agar and Read, 2002).

It was clear that where PSAMHS had occurred there was an institutional minimization of the impact and inadequate or non-existent policies in place to ensure that victims could continue to receive appropriate services (Kennedy, 2002; Hesketh, 2003). However, individuals who had their own personal connection to the issue felt that it would help them to respond well if a client disclosed PSAMHS.

The findings have potential implications for policy makers, service providers, managers, individual practitioners and educators. There are clearly wide ranging responses to incidents of PSAMHS at a macro level within the mental health system and this is probably not helped by different professions having different regulatory bodies; a different language to describe PSAMHS; and different expectations of registrants in terms of reporting colleagues. This is problematic and inconsistent with other imperatives to ensure multi disciplinary teams can provide co-ordinated and seamless services. However, this fragmentation is also evident in the theoretical location of the literature (Schoener, 1995; Melville-Wiseman, 2008). Legal and policy imperatives appear to have been partially effective in bringing some abusive professionals to account but they currently rely on the ability of victims, colleagues and employers to report cases once known. For victims this is not a 'quick fix' issue and for many understandable reasons it can take sometimes decades to disclose. To date, there is no evidence that regulatory bodies have developed processes to take account of this.

\section{Conclusions}

This study is limited in terms of only capturing the views of a small sample of interested participants. The findings should therefore be treated with caution. However, it does seem clear that further exploration of the institutional dimensions of PSAMHS would be useful.

Such corruptions of the task of a professional could be addressed by giving primacy to teaching and training on social inequalities, power dynamics and their role in PSAMHS in pre-registration and post qualifying training. This places an imperative on educators and trainers to impart skills in handling difficult conversations and being honest about the level of courage that care work requires. Notions of inter professional working focus on how different professionals can get on well for the sake of their shared clients - but such professionals also needs skills and courage to critically challenge each other in order to avoid colluding with abusive practice. This can be uncomfortable work. Some participants spoke about their anger and shame at what some colleagues had done but they had not found the courage to whistle blow. 
Putting the onus on individuals to come forward makes services unhealthy places to work and therefore unhealthy places to provide services. It is likely that there are a significant number of clients who have not yet disclosed PSAMHS, and therefore a significant number of professionals who have also not had opportunities to speak about their knowledge as witnesses. However, some courageous professionals do come forward (including in this study) and, often drawing on their own personal connection to abuse, have helped to bring the subject, albeit briefly, to the forefront.

This study is both small scale and limited in scope but points to the need for more robust studies into prevalence and impact as well as investigations into the quality of our mental health services and the experiences of the people who use them.

\section{References}

Acker, J. A. (1995) Alex's story. in J.C. Gonsiorek (Ed.), Breach of Trust: Sexual exploitation by health care professionals and clergy. London: Sage

Agar K., and Read J. (2002) What happens when people disclose sexual or physical abuse to staff at a community mental health centre? International Journal of Mental Health Nursing, $11,2,70-79$

Attride-Stirling, J. (2001) Thematic networks: An analytic tool for qualitative research. Qualitative Research, 1, 3, 385-405

Bates, C. M. and Brodsky, A. M. (1989) Sex in the Therapy Hour: A case of professional incest. New York: Guilford

Benowitz, M. (1995) Comparing the experiences of women clients sexually exploited by female versus male psychotherapists. in JC. Gonsiorek (Ed.). Breach of Trust: Sexual Exploitation by health care professionals and clergy. London: Sage

Biehal, N. (2005) Changing practice: Participation, rights and community care. British Journal of Social Work, 23, 443-458

Borys, D. and Pope, K. (1989) Dual relationships between therapist and patient: A national study of psychologists, psychiatrists and social workers. Professional Psychology: Research and Practice, 20, 5, 282-293

Brown, H. and Keating, F. (1998) 'We're doing it already ..': Adult protection in mental health services. Journal of Psychiatric and Mental Health Nursing, 5, 4, 273-280

Cambridge, P. (1999) The First Hit: a case study of the physical abuse of people with learning disabilities and challenging behaviours in a residential setting. Disability and Society, 14, 3, 285-308

Cambridge, P., Beadle-Brown, J., Mansell, J., Milne, A, and Whelton, B. (2006) Exploring the Incidence, Risk Factors, Nature and Monitoring of Adult Protection Alerts. Canterbury: Tizard Centre 
Cho J. and Trent, A, (2006) Validity in qualitative research revisited. Qualitative Research, 6, $319-340$

Colton, M. (2002) Factors associated with abuse in residential child care institutions. Children and Society, 16, 1, 33-44

Council for Healthcare Regulatory Excellence (2008) Learning about Sexual Boundaries between Healthcare Professionals And Patients: A report on education and training. London: Council for Healthcare Regulatory Excellence

Casemore, R. (2001) Surviving Complaints against Counsellors and Psyhcotherapists: Towards understanding and healing. Ross-on-Wye: PCCS Books

D'Addario, L. (1977) Sexual Relationships between Female Clients and Male Therapists. Unpublished doctoral dissertation. San Diego, CA: California School of Professional Psychology

Department of Health (2000) No Secrets: Guidance on developing and implementing multi-agency policies and procedures to protect vulnerable adults from abuse. London: Department of Health

Disch, E. and Wohlberg, J. (1995) The Boston Experience: Responding to sexual abuse by professionals. in J.C. Gonsiorek (Ed.), Breach of Trust: Sexual exploitation by health care professionals and clergy. London: Sage

Doel, M., Allmark, P., Conway, P., Cowburn, M., Flynn, M., Nelson, P. and Tod, A. (2009) Professional Boundaries. London: General Social Care Council

Fortune, M.M. (1992) Is Nothing Sacred? The story of a pastor, the women he sexually abused, and the congregation he nearly destroyed. New York: Harper Collins

Garrett, T., and Davis, J. (1994) Epidemiology in the UK. in D. Jehu (Ed.), Patients as Victims. Chichester: Wiley

Gartrell, N.K., Herman, J., Olarte S.,Feldstein M., and Localio R. (1987) Reporting practices of psychiatrists who knew of sexual misconduct by colleagues. American Journal of Orthopsychiatry, 57, 287-295

Gechtman, L. and Bouhoutsos, J. (1985) Sexual Intimacy between Social Workers and their Clients. Paper presented at the annual meeting of the Society for Clinical Social Workers. Universal City, California

General social Care Council (2009) Raising Standards Conduct Report 2003-2008. General Social Care Council. Available on-line at: http://www.gscc.org.uk/cmsFiles/Publications/ GSCC_Raising_Standards_SW_Conduct_03-08_Report.pdf [Accessed 25.1.12]

General social Care Council (2011) Professional Boundaries Guidance for Social Workers. General Social Care Council. Available on line at: http://www.gscc.org.uk/cmsFiles/ Conduct/GSCC_Professional_Boundaries_guidance_2011.pdf [Accessed 25.1.12]

Hawton, K., Simkin, S., Rue, J., Haw, C., Barbour, F., Clements, A., Sakarovitch, C., and Deeks, J. (2002) Suicide in female nurses in England and Wales. Psychological Medicine, 32, 239-250

Hesketh, W., (2003) Medico Crime: Time for a police-professions. protocol? The Police Journal, 76, 121-131

Hewitt, J. L. (2008) Dangerousness and mental health policy. Journal of Psychiatric and Mental Health Nursing, 15, 186-194 
Holroyd, J. and Brodsky, A. (1977) Psychologists' attitudes and practices regarding erotic and nonerotic physical contact with clients. American Psychologist, 32, 843-849

Hughes, R. and Huby, M. (2002) The application of vignettes in social and nursing research. Journal of Advanced Nursing 37, 4, 382-6

Jackson, C. (2006) Out of sight. Are trusts doing enough to prevent rape and sexual assault on psychiatric wards? Mental Health Today, 6, 7, 8-9

Jasper, M. (2005) Using reflective writing within research. Journal of Research in Nursing, 10, 3, 247-260

Kardner, S. H., Fuller, M., and Mensh, I. N. (1973) A survey of physicians' attitudes and practices regarding erotic and non-erotic contact with patients. American Journal of Psychiatry, 130, 10, 1077-1081

Kennedy, M. (2002) White collar crime: Vulnerable women, predatory clergyman. Journal of Adult Protection, 4, 4, 23-33

Melville-Wiseman, J. (2008) Pathologies or apologies: A case study of an incident of professional sexual abuse in the mental health system. University of Kent: Unpublished Doctoral Thesis

MIND (2004) Ward Watch: Mind's Campaign to Improve Hospital Conditions for Mental Health Patients. Mind. Available on-line at http://www.mind.org.uk/assets/0000/0353/ward_ watch_report.pdf [Accessed 25.1.12]

National Patient Safety Agency (2006). With safety in mind: mental health services and patient safety. Patient Safety Observatory Report 2. London: National Patient Safety Agency.

Nursing and Midwifery Council (2011) Annual Fitness to Practice Report 2010-2011. Nursing and Midwifery Council. Available on line at: http://www.nmc-uk.org/Documents/ Annual_reports_and_accounts/FTPannualReports/NMC-annual-fitness-to-practicereport-2010-2011.pdf [accessed 1st February 2012]

O'Brien, A. J. (2001) The therapeutic relationship: Historical development and contemporary significance. Journal of Psychiatric and Mental Health Nursing, 8, 129-137

Office of Public Sector Information (2003) Sexual Offences Act 2003. London: The Stationary Office

Penfold, P.S., (1998) Sexual Abuse by Health Professionals: A Personal Search for Meaning and Healing. Toronto: University of Toronto Press

Peterson, M. (1992) At Personal Risk: Boundary violations in professional-client relationships. New York: Norton

Pleming, N. (2005) The Kerr/Haslam Inquiry. London: The Stationary Office

Pope, K. S., Levenson, H. and Schover, L. (1979) Sexual intimacy in psychology training: Results and implications of a national survey. American Psychologist, 34, 8, 682-689

Repper, J. and Perkins, R. (2003) Social Inclusion and Recovery: A model for mental health practice. London: Baillière Tindall

Russell, J. (1993) Out of Bounds: Sexual exploitation in counselling and therapy. London: Sage

Schoener, G. R., Milgrom, J.H., and Gonsiorek, J. (1984) Sexual exploitation of clients by therapists. Women and Therapy, 3, 3-4, 63-69

Schoener, G. R. (1995) Historical overview. in JC. Gonsiorek (Ed.). Breach of trust: Sexual exploitation by health care professionals and clergy. London: Sage 
Simons, H., (2009) Case Study Research in Practice. London: Sage

Stanley, N., Manthorpe, J. and White, M. (2007) Depression in the profession: worker's experiences and perceptions. British Journal of Social Work, 37, 281-298

Stake, R. E. (1995) The Art of Case Study Research. London: Sage

Wardaugh, J. and Wilding, P. (1993) Towards an explanation of the corruption of care. Critical Social Policy, 13, 37, 4-31

Williams, J. (1996) Social inequalities and mental health: Developing services and developing knowledge. Journal of Community and Applied Psychology, 6, 311-316

Williams J. and Keating F. (2000) Abuse in mental health services: Some theoretical considerations. The Journal of Adult Protection, 2, 3, 32-39

Wohlberg, J.W., McCraith, D.B., and Thomas, D.R., (1999) Sexual misconduct and the victim survivor: A look from the inside out. in J.D. Bloom, C.C. Nadelson, and M.T. Notman, (Eds.) Physician Sexual Misconduct. American Psychiatric Press, Washington, D.C.

Yin, R.K. (1992) The Case Study as a Tool for Doing Evaluation. Current Sociology, 40, 1, 121-137

Yin, R.K. (2009) Case Study Research: Design and methods. Fourth Edition. London: Sage 